\title{
VUV absorption spectroscopy measurements of the role of fast neutral atoms in high-power gap breakdown
}
A. B. Filuk, J.E. Bailey, M. E. Cuneo, P. Lake, T. J. Nash, D. D. Noack, Sandia National Laboratories, Albuquerque, New Mexico, 87185

Weimann Institute of Science, Rehovot, Israel, 76100

The maximum power achieved in a wide variety of high-power devices, including electron and ion diodes, $\mathrm{z}$ pinches, and microwave generators, is presently limited by anode-cathode gap breakdown. A frequently-discussed hypothesis for this effect is ionization of fast neutral atoms injected throughout the anode-cathode gap during the power pulse. We describe a newly-developed diagnostic tool that provides the first direct test of this hypothesis. Time-resolved vacuumultraviolet absorption spectroscopy is used to directly probe fast neutral atoms with $1 \mathrm{~mm}$ spatial resolution in the $10 \mathrm{~mm}$ anode-cathode gap of the SABRE $5 \mathrm{MV}, 1 \mathrm{TW}$ applied-B ion diode. Absorption spectra collected during Ar RF glow discharges and with $\mathrm{CO}_{2}$ gas fills confirm the reliability of the diagnostic technique. Throughout the 50-100 ns ion diode pulses no measurable neutral absorption is seen, setting upper limits of $0.12-1.5 \times 10^{14} \mathrm{~cm}^{-3}$ for ground state fast neutral atom densities of $\mathrm{H}, \mathrm{C}, \mathrm{N}, \mathrm{O}, \mathrm{F}$. The absence of molecular absorption bands also sets upper limits of $0.16-1.2 \times 10^{15} \mathrm{~cm}^{-3}$ for common simple molecules. These limits are low enough to rule out ionization throughout the gap as a breakdown mechanism. This technique can now be applied to quantify the role of neutral atoms in other high-power devices. 


\section{DISCLAIMER}

This report was prepared as an account of work sponsored by an agency of the United States Government. Neither the United States Government nor any agency thereof, nor any of their employees, make any warranty, express or implied, or assumes any legal liability or responsibility for the accuracy, completeness, or usefulness of any information, apparatus, product, or process disclosed, or represents that its use would not infringe privately owned rights. Reference herein to any specific commercial product, process, or service by trade name, trademark, manufacturer, or otherwise does not necessarily constitute or imply its endorsement, recommendation, or favoring by the United States Government or any agency thereof. The views and opinions of authors expressed herein do not necessarily state or reflect those of the United States Government or any agency thereof. 


\section{DISCLAIMER}

Portions of this document may be illegible in electronic image products. Images are produced from the best available original document. 


\section{Introduction}

Pulsed power devices generate TW-levels of electrical power for a variety of applications. Despite achieving 100-TW power levels, almost all pulsed power devices are limited by loss of electrical insulation across an anode-cathode gap at some time during the power pulse. This problem is common to many high-power devices, including $\mathrm{z}$ pinches, microwave tubes, Magnetically Insulated Transmission Lines (MITLs), and electron and ion beam diodes. Understanding the mechanisms and mitigating gap breakdown offers the promise of reaching a higher-power regime. One hypothesis for gap breakdown is that neutral atoms desorbed from the anode surface participate in charge-exchange with accelerated ions and the resulting fast atoms rapidly fill the anode-cathode ( $\mathrm{AK}$ ) region. In this context, "fast" means the atoms cross the gap on the time scale of the pulse, typically implying $>10 \mathrm{~cm} / \mu \mathrm{sec}$ velocities. If these fast neutral atoms are of sufficient density and can be ionized during the power pulse, the resulting extra charge flow causes the voltage across the anode-cathode gap to rapidly drop. In this paper we describe spectroscopic measurements showing that this mechanism is not responsible for gap breakdown in a 1 TW ion diode.

There has been considerable speculation, some calculations, and few direct measurements of neutral atoms in high power devices. Prono et al. recorded weak continuum light across an ion diode gap [1] and, presuming that the light originated from neutral atom emission, they constructed a model for gap breakdown by ionization of dense, fast neutral atoms. Subsequently, ionization of charge exchange neutral atoms was used as the foundation of a semi-empirical gap closure model [2] and proposed as a fundamental limitation on powers attainable in magnetically-insulated transmission lines (MTTLs). This suggestion provided the initial motivation for the experiments described in this paper. 
In later experiments, $\mathrm{Pal}$ and Hammer [3], and Maron et al. [4] both measured $\mathrm{H}_{\beta}$ emission in an ion diode anode plasma. It is important to recognize the distinction between anode plasma measurements and AK gap measurements. Anode plasma measurements are valuable and may be related to AK gap behavior, but they are not a direct means of characterizing the AK gap itself. Nevertheless, in Ref. [4] the initial $\sim 1 \mathrm{~cm} / \mu$ s plasma advance was modeled by fast-neutral ionization, but this plasma advance appeared to stop early in the power pulse and thus could not explain the gap breakdown. Litwin and Maron [5] then constructed a more-detailed neutral chargeexchange expansion model for an anode plasma and found that rapid ionization required neutral densities of $\sim 410^{5} \mathrm{~cm}^{-3}$. Bluhm et al. [6] mention measurements of a neutral flux about double the ion flux throughout a $\sim 800 \mathrm{~ns}, 0.5 \mathrm{MV}$ ion beam pulse, using secondary electron detectors and a charge-exchange ceil with an ion detector. Horioka et al. [7] report uncalibrated shadowgraphy and resonant interferometer measurements across a diode AK gap, showing indications of neutral atoms in the gap $\sim 150 \mathrm{~ns}$ after the start of diode current. Interferometer measurements on a diode by Tuszewski et al. [8] showed no indication of plasma forming in the diode during the $0.6 \mu$ s ion beam pulse, but did exhibit an interesting negative phase shift several $\mathrm{mm}$ from the anode at about the time of gap breakdown; they noted that this phase shift could be produced by $\sim 10^{16} \mathrm{~cm}^{-3}$ neutral atoms in the probed volume. In all of these investigations the fast-neutral atom anodecathode gap density estimates required to explain the phenomena were on the order of $10^{15}$ $10^{17} \mathrm{~cm}^{-3}$.

In recent ion diode experiments conducted at the Particle Beam Fusion Accelerator II (PBFA II) and SABRE accelerators, the limits on the achievable ion beam power have been attributed [9] to some form of gap breakdown. In these applied-B ion diodes [10] a strong applied magnetic field (typically several Tesla) is used to restrict electron flow across the anode-cathode gap when multi- 
MV voltages are applied. This enables the accelerated ion beam to carry a large fraction of the current across the gap. The intense ion beam can be extracted and used as an inertial confinement fusion driver [11], for example. The 20-TW, 2-cm AK gap PBFA II and the 1-TW, 1-cm AK gap SABRE diodes are among the highest power ion beam devices yet tested and have been described in detail elsewhere $[10,12]$. In the computational work of Pointon [13], calculations of chargeexchange effects in ion diodes showed that for PBFA II conditions the contribution of electron or proton impact ionization of neutral atoms to the diode current would be small unless the neutral atom density approached $-10^{15} \mathrm{~cm}^{-3}$, a conclusion similar to previous studies in low power diodes. It was found that, in the PBFA II diode, generation of such a $\sim 10^{15} \mathrm{~cm}^{-3}$ charge exchange neutral atom density could occur in the $\sim 40 \mathrm{~ns}$ pulse if they assumed ad hoc an initial $10^{17} \mathrm{~cm}^{-3}$ layer of cold neutrals in the $2 \mathrm{~mm}$ region near the anode. The effect of charge exchange on diode voltagecurrent characteristic curves was also investigated theoretically in Ref. [14]. A possible mechanism for the production of fast (charge-exchange) neutral atoms with the field-threshold, LiF thin-film ion source [15] used in PBFA II and SABRE experiments was described in Ref. [16-18]. In this scenario a cold impurity layer is formed by electron thermal and stimulated desorption of adsorbed impurities due to the hundreds of $\mathrm{A} / \mathrm{cm}^{2}$ electron leakage current across the magnetic insulation to the anode surface. Expansion and ionization of these neutral atoms may be responsible for the sharp rise in beam impurity ions at the time of rapidly-dropping voltage $[9,16-18]$. This localized, nearanode neutral ionization scenario cannot produce gap breakdown by itself because the current drawn is still subject to the space charge limit. However, such a mechanism could lead to filling of the gap with fast charge exchange neutral atoms that might subsequently contribute to breakdown.

The results described above provide cause for concern that neutral ionization leads to gap breakdown. Quantitative evaluation of this hypothesis requires direct measurements of the neutral 
density in the gap between the anode (or anode plasma) and the cathode. Prior to the present study, the only such measurements were Li I visible-emission spectra obtained $[19,20]$ on the PBFA II and SABRE ion diodes. These spectra showed that less than $2 \times 10^{12} \mathrm{~cm}^{-3}$ fast $(-50 \mathrm{~cm} / \mu \mathrm{s}) \mathrm{Li}$

neutral atoms exist in the AK gap of these diodes, far lower than the approximately $10^{15} \mathrm{~cm}^{-3}$ densities estimated to be needed for fast gap breakdown. However, visible spectroscopy cannot search effectively for many common neutral atoms such as $\mathrm{H}, \mathrm{C}$, and $\mathrm{O}$ because the resonance transitions lie in the vacuum ultraviolet (VUV) and the excited states leading to transitions in the visible may not be populated. In this study, we use a pulsed VUV continuum backlighter source shining between the SABRE diode anode and cathode to spectroscopically measure the groundstate neutral atom density. The backlighter emission is viewed with a time-resolved VUV spectrometer [21]. The absorption of the backlighter continuum at characteristic wavelengths of the resonance transitions (or edges) gives a direct absolute measurement of the line-integrated AK gap neutral atom (or molecule) density. In these experiments no measurable absorption features were seen, setting upper limits of $0.1-2 \times 10^{14} \mathrm{~cm}^{-3}$ for ground state densities of $\mathrm{H}, \mathrm{C}, \mathrm{N}, \mathrm{O}$, and F. Similar upper limits for common simple molecules are $0.16-1.2 \times 10^{15} \mathrm{~cm}^{-3}$. These bounds are sufficiently low to rule out ionization of fast neutral atoms throughout the gap as a mechanism leading to gap breakdown. Ionization of molecules is also very unlikely to cause gap breakdown, but we cannot preclude the possibility that electrons produced by molecule ionization perturb the gap potential and contribute indirectly to breakdown.

\section{VUV Absorption Diagnostic}

The absorption spectroscopy diagnostic system consists of a VUV continuum source and a time-resolved normal-incidence VUV spectrograph. The continuum backlighter is a relatively-long 
pulsed discharge known as a BRV source [22]. The BRV generates a 100-200 ns wide pulse of -500-1500 § light from a 3-mm-diameter aperture. The light is directed across the AK gap parallel to the anode and into the VUV spectrograph as shown in Figure 1. The spectrometer line-of-sight collection region is defined by an elliptical input mirror, focussing to a $\sim 1 \mathrm{~mm}$ waist in the AK gap dimension (i.e., in the direction of ion beam acceleration), but not imaging in the vertical direction [21]. The emission from the BRV that is collected by the spectrometer input mirror samples a $1 \mathrm{~cm}$ tall region at the AK gap, in the middle of the active anode region. The active anode inner and outer radii are $4.5 \mathrm{~cm}$ and $6.5 \mathrm{~cm}$, and the chord length for absorption in front of the anode is about $7 \mathrm{~cm}$. Note that the elliptical mirror can also collect light emitted from excited atoms and ions in the diode gap region; the emission collection volume is larger than the absorption collection volume (see Figure 1).

The spectrograph is a $1 \mathrm{~m}$ focal length instrument with input slit widths of $60-200 \mu \mathrm{m}$. The $120 \mu \mathrm{m}$ slit width used in these experiments corresponded to $\sim 2 \AA$ instrument width at the detector plane with a $600 \mathrm{1} / \mathrm{mm}$ grating. The spectral range was $\sim 600-1250 \AA$, spanning the resonance lines and photoionization energies of $\mathrm{H}, \mathrm{C}, \mathrm{N}, \mathrm{O}$, and $\mathrm{F}$ as shown in Table 1 . These were the atoms most likely to be present in the diode gap during the power pulse.The detector is a 40-mm-diameter gated micro-channel plate (MCP) framing camera. We use a CuI photocathode in a seven stripline configuration. The striplines are gated on with 6-ns-wide $1000 \mathrm{~V}$ pulses spaced 8 ns apart, providing time-resolved data spanning a $54 \mathrm{~ns}$ total time record. The spectra at the MCP framing camera output are recorded on TMAX P3200 film.

The time-resolved spectrograph has been used to record emission spectra from the SABRE anode and cathode plasmas [23] and there is no reason to suspect the operation fails in an absorption mode. Nevertheless, we performed two tests to verify that the absorption system worked 
as intended. Such tests are not trivial because, as described above, detectable absorption features require a medium with Doppler broadening that is comparable to the instrument resolution.In the first test, a spectrum was collected when a $6 \mathrm{mTorr}$ Ar RF glow discharge was present in the SABRE vacuum chamber. The Ar I ionization edge at $787 \AA$ (M-shell) was clearly seen as a step in the continuum intensity on film. Since the glow is only very weakly ionized, we estimate the ArI ground state population density over the $\sim 150 \mathrm{~cm}$ path as $N=N_{o} p T_{d}\left(p_{o} T\right) \sim 2.0 \times 10^{14} \mathrm{~cm}^{-3}$, where $N_{o}$ is Loschmidt's number. The uncertainty in this estimate is $\pm 30 \%$, due mainly to the fact that a differentially-pumped gas flow was used, rather than a static gas fill. The ratio of the light intensity above background fog on the film just to the red side of the $787 \AA$ edge, $I_{+}$, to that on the blue side, $I_{-}$, was $I_{+} I_{-}=4.9 \pm 3.4$, with a large uncertainty because of the near-fog film density on the blue (strongly absorbed) side of the edge. Using the published average absorption coefficients (assuming the one-dimensional slab approximation for radiation transport) to the red and blue sides of $\mu_{+}=30 \mathrm{~cm}^{-1}$ and $\mu_{-}=800 \mathrm{~cm}^{-1}$ respectively [24], one has a theoretical intensity ratio

$$
\frac{I_{+}}{I_{-}}=e^{\left(\mu_{-}-\mu_{+}\right) \frac{N}{N_{o}} l}=2.4 \pm 0.7
$$

which is within the uncertainty of our observed intensity ratio. Thus, for an absorption edge the diagnostic works as expected. The blue-side absorption coefficients $\mu_{\text {_ }}$ for various neutral atoms are shown in Table 1 ; one typically has $\mu_{+}<<\mu_{-}$such that $\mu_{+} N L / N_{o}$ may be neglected.

A second test of the absorption system was to detect the $\mathrm{CO}_{2}$ molecular absorption spectrum, particularly the two narrower bands at about $1090 \AA$ and $1125 \AA$ that have shapes similar to Doppler-broadened atomic lines. A $5.5 \mathrm{mTorr}$ differentially-pumped $\mathrm{CO}_{2}$ gas fill gave the absorption spectrum shown in Figure 2. Overlaid is a spectrum calculated from the work of Chan et al. [25], using here a line-density $N l=3.1 \times 10^{16} \mathrm{~cm}^{-2}$ corresponding to an absorption path 
length of $160 \mathrm{~cm}$ at $5.5 \mathrm{mTorr}$. Our spectrum shows more complicated structure than indicated by Chan et al's $\mathrm{CO}_{2}$ absorption spectrum, possibly due to improved spectral resolution in the present experiments. The structure does not appear to be due to BRV plasma impurity emission lines superimposed on the continuum since the no-absorption spectrum does not exhibit this structure. Note that the no-absorption spectrum in Figure 2 has been smoothed, and the signal is rapidly falling off below $890 \AA$ since this region was near the edge of the MCP. Overall, the agreement with Chan et al is reasonable.

\section{Results}

We collected absorption spectra for several SABRE shots with the line-of-sight at various $z$ locations across the $10 \mathrm{~mm}$ AK gap: 0-1 mm, 1-2 mm, and 5-6 mm from the anode. Figure 3 shows typical current waveforms and VUV spectrometer frame center-times for one such shot. Note that in these experiments impurity ions that could originate from ionization of contaminant neutral atoms in the gap appear in the ion beam at roughly $27 \mathrm{~ns}$, where $t=0$ is approximately the beginning of the ion beam current. Despite several shots with good continuum intensity on film, no absorption lines were seen at all, even later in the pulse (t $-50-150$ ns in Figure 3).The absence of absorption lines with our given signal-to-noise ratio ( $\mathrm{S} / \mathrm{N}$ ) establishes upper bounds on the AK gap ground state neutral densities for the various atoms listed in Table 1. As well, the absence of absorption bands of known strength and wavelengths gives upper bounds on various molecular species that might be present.

In addition to the continuum light, numerous intense ion lines were seen in emission at various distances from the anode during the power pulse. When viewing $0-1 \mathrm{~mm}$ from the anode, lines 
from various ion charge states of $\mathrm{C}, \mathrm{O}$, and $\mathrm{F}$, including $\mathrm{C} \mathrm{IV}, \mathrm{O}$ VI, and F VII appeared just after the start of the ion beam. These lines indicate plasma formation near the anode during the pulse, and again confirm that the spectrograph was functioning as intended. The emission was also detected later in the pulse with the line-of-sight at various distances from the anode. This may simply be undesired light collected from the very-bright anode plasma, since the quality of the elliptical collecting optic allows some stray light from the anode region to enter the spectrograph even when the nominal spatial position is located in the middle of the gap. Alternatively, it may actually indicate plasma jetting into the diode gap. This plasma would have to be emitted nonuniformly from the anode or cathode since simultaneously-acquired space- and time-resolved visible spectra show Stark-shifted $\mathrm{Li}$ I emission, indicating that a large electric field exists in at least some regions along the line of sight. Such large fields are incompatible with plasma densities above about $5 \times 10^{13} \mathrm{~cm}^{-3}$ since the plasma would screen the field. While the appearance of highlyionized plasma ions is interesting, it is beyond the focus of this report.

In order to see an absorption line, the Doppler-broadened line width should be comparable to the instrument spectral resolution. If the line width is narrower than the spectral resolution, the absorption feature is smeared out over the instrument profile. The narrower the line width is, the higher the density must be in order to detect an absorption feature. In the absence of strong reemission effects, for a spectral line of Lorentzian (natural) width $\Delta \lambda_{L}$ and Doppler width $\Delta \lambda_{G}$ one may write the transmitted light intensity $I(\lambda)$ of incident continuum light $I_{0}(\lambda)$ across a uniform attenuating medium of path length 1 and absorption coefficient $k(\lambda)$ as [26]

$$
\frac{I(\lambda)}{I_{0}(\lambda)}=e^{-k(\lambda) l}
$$

where, using $\alpha \equiv \ln 2, a \equiv \alpha \Delta \lambda_{L} / \Delta \lambda_{G}$, and $s(\lambda) \equiv 2 \alpha\left(\lambda-\lambda_{o}\right) / \Delta \lambda_{G}$ one has 


$$
\frac{k(\lambda)}{k_{0}}=\frac{a}{\pi} \int_{-\infty}^{\infty} \frac{e^{-y^{2}} d y}{a^{2}+(s(\lambda)-y)^{2}}
$$

Here, $k_{0} l$ is the optical depth at line center,

$$
k_{o}=\frac{2 \lambda_{o}^{2}}{\Delta \lambda_{G}} \sqrt{\frac{\alpha}{\pi}} \frac{\pi e^{2}}{m_{e} c^{2}} N f
$$

with $N$ absorbers per unit volume of absorption oscillator strength $f$. In this equation we neglect the stimulated emission term, since we expect the upper levels of the resonance lines to be much less populated than the ground states. This is justified by the fact that spontaneous emission from the neutral atom resonance lines is not observed, implying that the upper levels are not sufficiently populated to contribute significantly in Eqn. 3.

For $a \leq 0.01$ one has the limit of small natural damping, which should hold in our case of VUV resonance lines even for Doppler broadening effective temperatures $T_{D}$ as low as room temperature. Then (2) can be written as [26]

$$
\frac{k(\lambda)}{k_{0}}=e^{-s^{2}}-\frac{2 a}{\sqrt{\pi}}[1-2 s F(s)]
$$

where $s$ is as above and

$$
F(s) \equiv e^{-s^{2}} \int_{0}^{s} e^{y^{2}} d y
$$

The absorption depends on the value of the line-center optical depth, $\mathrm{k}_{\mathrm{o}} \mathrm{l}$, which is proportional to the number of absorbers in the line-of-sight. We average (1), using (4), over the region $\Delta \lambda_{i}$ centered at $\lambda_{\mathrm{o}}$ for each line of interest in Table 1 , given a Doppler width $\Delta \lambda_{\mathrm{G}}$ (FWHM) as specified by $\mathrm{T}_{\mathrm{D}}$

$$
\Delta \lambda_{G}=2 \lambda_{o} \sqrt{\frac{2 \alpha T_{D}}{A m_{p} c^{2}}}
$$


Here, $T_{D}$ is defined as the "effective" Doppler broadening temperature, in the sense that it represents the width of the velocity distribution parallel to the anode, even though the actual velocity distribution may not be characterized by a Maxwellian distribution.

An upper bound on the $\Delta \lambda_{i}$-average fractional absorption $1-</ I_{0}>$ is the reciprocal of the signal to noise ratio, $1 /(\mathrm{S} / \mathrm{N})$. This in turn gives, using (1)-(4), an upper bound on the density $N$. Thus, for a given $\mathrm{S} / \mathrm{N}$ one may calculate the upper bounds on the ground state density $N$ as a function of $T_{D}$ for each resonance line in our spectral window. Results from such a calculation are shown in Figure 4 for our parameters of $S / N \approx \sigma$ and $\Delta \lambda_{i}=2 \AA$. Note that at low $T_{D}$ the upper bound on ground state neutral density rises rapidly due to the smearing effect of the instrument width masking the absorption feature. We have used the fractional absorption averaged over the larger of $\Delta \lambda_{i}$ and $\Delta \lambda_{G}$, as this is what is actually measured in an experiment. The horizontal portions of the curves in Figure 4 are in the region $\Delta \lambda_{G} \gg \Delta \lambda_{i}$.

In general, the velocities responsible for producing the Doppler broadening are not known. However, in previous experiments [19],we measured the parallel and perpendicular velocity of fast Li I neutrals produced by charge exchange of the accelerated lithium ion beam. In the present analysis we assume that the effective Doppler broadening temperature for all fast neutral atoms is the same as previously measured for $\mathrm{Li}$ I charge exchange neutral atoms. The implications of this assumption are discussed below. In the previous $\mathrm{Li}$ I measurements we found $\mathrm{T}_{\mathrm{D}} \sim 3.3 \mathrm{keV}$. In this case, the Doppler FWHM is comparable to the instrument width even for heavier impurities like oxygen. From the intersection of the curves in Figure 4 with the dashed line at $3.3 \mathrm{keV}$ and using the $7 \mathrm{~cm}$ pathlength, we obtain the upper bounds for the fast ground-state neutral densities shown in Table 2. The detection of molecular absorption is insensitive to Doppler broadening, since 
molecular features are generally much broader than the instrument resolution (e.g., Fig.2). The upper bounds for various molecular species are shown in Table 3.

\section{Discussion}

No absorption features were observed in these experiments. The negative nature of this result forces us to make assumptions about the line width, or equivalently the effective Doppler temperature $T_{D}$, during the analysis. If the neutrals are produced before the power pulse, then the velocity both parallel and perpendicular to the anode can be small and the minimum detectable density could be large. However, the goal of this work is to search for fast neutral atoms, where fast means they fill the gap on the time scale of the pulse. Neutral atoms that have large velocities perpendicular to the anode tend to also have large velocities parallel to the anode. Thus, it is helpful to express $T_{D}$ in terms of the velocity measured perpendicular to the anode $v_{\perp}$ and the half-angle divergence $\Delta \theta=v_{p} / v_{\perp}$, where $v_{p}$ corresponds to the half width at half maximum of the velocity distribution parallel to the anode. Then $\Delta \lambda_{\mathrm{G}}=2 \lambda_{0} \mathrm{v}_{\mathrm{p}} / \mathrm{c}$ and

$$
T_{D}=\left(\frac{v_{p}}{c}\right)^{2} \frac{A m_{p} c^{2}}{2 \alpha}=\frac{A m_{p}\left(v_{\perp}\right)^{2}}{2 \alpha}(\Delta \theta)^{2}=\frac{E_{\perp}}{\alpha}(\Delta \theta)^{2}
$$

, where $E_{\perp}$ is the fast neutral atom energy in the direction perpendicular to the anode. Our definition of fast neutral atoms implies that a minimum value of $v_{\perp}$ is approximately $\mathrm{v}_{\perp}{ }^{\min } \sim 0.8 \mathrm{~cm} / 40 \mathrm{~ns}=2 \times 10^{7} \mathrm{~cm} / \mathrm{sec}$. A lower limit for $T_{D}$ then depends mainly on $\Delta \theta$, the divergence of the fast neutral atoms.

In previous measurements of fast lithium neutral atoms [20] we found $v_{p}{ }^{L i} \sim 2.5 \times 10^{7} \mathrm{~cm} / \mathrm{sec}$, $\mathrm{v}_{\perp}^{\mathrm{Li}} \sim 5 \times 10^{7} \mathrm{~cm} / \mathrm{sec}, \mathrm{E}_{\perp}^{\mathrm{Li}} \sim 9.1 \mathrm{keV}, \Delta \theta^{\mathrm{Li}} \sim 0.5$, and $\mathrm{T}_{\mathrm{D}}^{\mathrm{Li}} \sim 3.3 \mathrm{keV}$. The upper bounds on the fast neutral atom density $\mathrm{N}$ displayed in Table 2 assumed that the contaminant atoms we are searching for here all have the same value of $T_{D} \sim 3.3 \mathrm{keV}$. According to the equation above, this 
assumption is satisfied if the contaminants are born with energy and divergence similar to the previous Li I measurements.

It is possible that $T_{D}$ for some fast neutral atoms is lower than for others, for example because they might be born in a different anode region. In order for this possibility to impact our basic conclusion that breakdown throughout the gap due to neutral ionization does not occur, $T_{D}$ must be much lower. Previous work $[5,13]$ and our own estimates below indicate that breakdown throughout the gap requires $\mathrm{N}_{\mathrm{f}} \sim 10^{15} \mathrm{~cm}^{-3}$. We now examine the quantitative implication for $\mathrm{H}$ and $F$, the lightest and heaviest contaminants considered here. From Fig. 4 , if $N_{f} \sim 10^{15} \mathrm{~cm}^{-3}$ for hydrogen, we require $T_{D}{ }^{H} \sim 1.3 \mathrm{eV}=(1 / 2540) T_{D}{ }^{\mathrm{Li}}$. In addition, the minimum velocity needed to qualify as a "fast" neutral atom implies that $\mathrm{E}_{\perp}{ }^{\mathrm{H}} \sim 208 \mathrm{eV}, 44$ times lower than $\mathrm{E}_{\perp}{ }^{\mathrm{Li}}$. At this minimum $v_{\perp}$, if $T_{D}{ }^{H} \sim 1.3 \mathrm{eV}$ we must have $\Delta \theta^{\mathrm{H}} \sim 0.065,7.6$ times lower than $\Delta \theta^{\mathrm{Li}}$. Similarly, if $N_{f} 10^{15} \mathrm{~cm}^{-3}$ for fluorine, we require $T_{D}{ }^{F} \sim 60 \mathrm{eV}=(1 / 55) T_{D}{ }^{L i}$. The minimum $v_{\perp}$ corresponds to $E_{\perp} \sim 3.96 \mathrm{keV}=(1 / 2.3) \mathrm{E}_{\perp}{ }^{\mathrm{Li}}$ and, if $\mathrm{T}_{\mathrm{D}}{ }^{\mathrm{F}}=60 \mathrm{eV}, \Delta \theta^{\mathrm{F}} \sim 0.010$, about 5 times lower than $\Delta \theta^{\mathrm{Li}}$.

To evaluate whether such reduced values of $T_{D}, E_{\perp}$, and $\Delta \theta$ are plausible it is necessary to invoke a model for how $E_{\perp}$ and $\Delta \theta$ arise. The only process to our knowledge that is capable of producing such large values of $E_{\perp}$ is charge exchange of accelerated contaminant ions within a cold neutral layer in front of the anode. The fast contaminant neutral atoms that result are expected to acquire the velocity and divergence of the ions at the instant the charge exchange takes place, as described in Ref. 20. Then $E_{\perp}$ depends on the spatial profile of the charge exchange probability as the ions are accelerated away from the anode. This probability in turn depends on the energy dependence of the charge exchange cross section and the spatial dependence of the cold layer density that causes the charge exchange. The cross section peaks at different energies for different ions. However, the previous $\mathrm{Li}$ I measurements [20]showed that for these conditions, the charge 
exchange occurs before the ion reaches the energy corresponding to the maximum charge exchange cross section. In this case the cross section is monotonically increasing for all ions as they are accelerated through cold layer. It is then reasonable that $E_{\perp}$ is similar for all ions, if they are accelerated through the same potential drop and the same cold layer that induces the charge exchange. Therefore, it is very unlikely that $\mathrm{E}_{\perp}{ }^{\mathrm{H}}$ could be 44 times lower than $\mathrm{E}_{\perp}^{\mathrm{Li}}$. On the other hand, a value of $E_{\perp}{ }^{F} \sim(1 / 2.3) E_{\perp}{ }^{L i}$ is possible.

The divergence of the fast neutral atoms is expected [20] to be comparable to, or slightly larger than, the divergence of the accelerated ions at the instant the charge exchange occurs. Note that this divergence is generally much larger than the final divergence of the accelerated ion beam, since the beam ions acquire much less energy parallel to the anode than perpendicular to the anode as they are accelerated across the bulk of the gap. This divergence results mostly from electric fields generated parallel to the anode, for example because of ion source non-uniformities or instabilityinduced field fluctuations. If $\Delta \theta$ is 4.9-7.6 times smaller than for $\mathrm{Li}$ we would have to suppose that the divergence inducing fields were smaller by similar amounts. We regard this as unlikely, since the divergence-inducing fields exist throughout the diode gap and the observed ion beam divergence varies by less than a factor of two from one species to another $[9,10]$. Our conclusion is that values of $T_{D}$ low enough to be consistent with $N_{f} \sim 10^{15} \mathrm{~cm}^{-3}$ are unlikely.

Assuming the upper bounds of Table 2 apply across the gap we can now show that impact ionization of neutral atoms is much too slow a mechanism to explain AK gap breakdown. We will be extremely conservative and use the maximum possible rates for electron impact ionization, rather than the $\mathrm{MeV}$ energies expected to actually populate the gap, as well as the maximum possible densities in Table 2. Note that ion impact ionization cross-sections are in general very similar to those of electrons, but attain their maxima at higher energies (typically of order 
$100 \mathrm{keV} / \mathrm{amu})$. The characteristic rate $1 / \tau$ for electron impact ionization avalanche may be written

$$
\frac{1}{\tau}=\frac{N n_{e} \sigma v}{n_{i}}=N \sigma v
$$

where we will use the maximum rates at $100-200 \mathrm{eV}$ electron energies of $\sigma v=(4,12,11,11,11) \times 10^{-}$ ${ }^{17} \mathrm{~cm}^{3} / \mathrm{ns}$ for $(\mathrm{H}, \mathrm{C}, \mathrm{N}, \mathrm{O}, \mathrm{F})$ respectively, as given in Lotz [27], to allow for a secondary electron avalanche. These give characteristic avalanche times $\tau$ for $(\mathrm{H}, \mathrm{C}, \mathrm{N}, \mathrm{O}, \mathrm{F})$ of $(2100,233,564,57$, 82) ns. The avalanche-ionized contaminant beam current density for our $\sim 50 \mathrm{~ns}$ pulses is estimated by $J_{c} \approx\left(q \bar{n}_{e} d\right) / \tau$. Using $\mathrm{n}_{\mathrm{e}} \sim 10^{13} \mathrm{~cm}^{-3}, \mathrm{~d} \sim 1 \mathrm{~cm}$, and $\tau=100 \mathrm{~ns}$, this gives $\mathrm{J}_{\mathrm{c}} \sim 0.016 \mathrm{kA} / \mathrm{cm}^{2}$, compared to measured ion current densities of $0.5-1.0 \mathrm{kA} / \mathrm{cm}^{2}$. Thus, we conclude that impact avalanche ionization of these AK gap neutral atoms is too slow to significantly affect diode behavior.

The upper bounds for molecular species given in Table 3 are approximately an order of magnitude higher than the bounds on neutral atoms. Consequently, it is more difficult to rule out participation of molecules in gap breakdown. For example, suppose the $\mathrm{H}_{2}$ density equals the upper bound established here $\left(1.2 \times 10^{15} \mathrm{~cm}^{-3}\right)$. We estimated the ionization time scale using a cross section of $8 \times 10^{-18} \mathrm{~cm}^{2}$ for primary ionization by the ion beam, assuming the ion current density rises to $1 \mathrm{kA} / \mathrm{cm}^{2}$ over $50 \mathrm{nsec}$, and also assuming the maximum cross section for secondary electron ionization. This over-estimates the ionization, since, in reality, the secondary electrons are quickly accelerated to high energies by the large electric field. With these assumptions, we found that the ionization fraction reaches $0.1 \%$ in about $20 \mathrm{nsec}$ and $1.0 \%$ in $50 \mathrm{nsec}$. These values imply that ionization of a $10^{15} \mathrm{~cm}^{-3} \mathrm{H}_{2}$ cloud is unlikely to cause catastrophic gap breakdown, but that the acceleration gap potential profile may be perturbed by the $\sim 10^{12} \mathrm{~cm}^{-3}$ electrons produced when 
the ionization fraction reaches $0.1 \%$. These estimate indicate the need for detailed computer simulations to evaluate the effect of molecular ionization. No such simulations are available at present for SABRE conditions. However, simulations have been performed [28] for conditions in the 20 TW diode used on the PBFA II accelerator. In Ref. [28] ionization of $5 \times 10^{15} \mathrm{~cm}^{-3} \mathrm{H}_{2}$ was sufficient to perturb the gap and modify the extracted ion current, but it did not cause gap breakdown. Note that this density is five times higher than the upper bound established here and that the ion current growth in the higher power PBFA II diode is faster, both factors that promote faster ionization. Our conclusion is that if a molecular cloud exists in the SABRE diode at the maximum density compatible with the present measurements, then it could contribute to degraded diode performance, but it is unlikely to cause gap breakdown by itself.

Note that the upper bounds of Table 2 and Table 3 are on the line-of-sight average densities. It is possible that narrow 'fingers' of higher densities could exist over some fraction $1 / \mathrm{p}$ of the absorption path with negligible absorption in between the fingers, making the neutral density upper bounds $\mathrm{p}$ times higher than the values in Table 2 . However, the large angular velocity spread of the charge-exchanged neutral atoms with typical ion source parameters will tend to merge any 'fingers' of neutral density out in the diode gap. Let the half-angle of the velocity spread of the charge-exchanged neutral atoms be $\theta$. The present experiments provide no new information on $\theta$, but in previous measurements[20] we found $\theta \sim 24^{\circ}$ for charge-exchanged $\mathrm{Li}$ I atoms. Using this as a guide, we can estimate limits on the size that such fingers might have. In order for the density upper bounds to be a factor of, say, $p=10$ times higher than in Table 2, we imagine the neutral atoms originate from spots of size $d$ separated by distance $D$. For an absorption measurement parallel to the anode a distance $x$ away from the surface, one then would require the neutral atoms to occupy at most $1 / p$ of the line-of-sight path, so that (in two dimensions) 


$$
\frac{d+2 x \tan \theta}{D}=\frac{1}{p}
$$

We don't know a priori either $D$ or the fractional spot size $\alpha \equiv d / D$. However, it is clear from (5) that $\alpha p<1$. Solving (5) for $D$ gives

$$
D=p \frac{2 x \tan \theta}{1-\alpha p}
$$

A lower bound for $D$ is obtained by neglecting $\alpha p$ in (6). Then, using $x=5 \mathrm{~mm}$ and the representative values from above, $D$ is at least $4.5 \mathrm{~cm}$. Note that this is very conservative, since these bounds would allow the neutral spots to fully merge at $x=5 \mathrm{~mm}$. Taking these estimates together with measurements of the ion beam that indicate it is uniform on this length scale, it seems that one may preclude the possibility of neutral atom fingers resulting from ion charge exchange.

As described above, it has been observed in previous experiments and simulations $[9,16-18]$ that the $\mathrm{Li}$ II component in the extracted ion beam is replaced by other sources of ion current at the end of the pulse. This work shows that these sources do not include significant current from neutralatom ionization in the bulk of the gap. However, neutral-atom ionization within hundreds of microns of the anode surface, as pointed out in References 16-18, could still contribute significant non-LiIl ions accelerated in the gap. This should not cause gap breakdown, but the accelerated ions can effectively shunt the Li II ion current. In the present work we did not attempt to study the neutral atom densities within $<0.5 \mathrm{~mm}$ of the anode surface.

The main value of this work is in the development of a method suitable for answering an important question for high power systems. So far, no fast neutral atoms or molecules have been detected and the data are not consistent with the hypothesis advanced by VanDevender that gap breakdown results from ionization throughout the gap. However, further measurements with improved signal to noise are needed to provide more definitive results on the role of molecules. 
Also, note that VanDevender's hypothesis may still be important in other systems. The production of fast neutral atoms in high-voltage gaps depends on various parameters (neutral-atom-layer formation over the electrodes, current densities, and electric fields) and thus can play different roles in different systems. In particular it may turn out to be important in the extremely high power systems currently being developed. The method developed here may be applied to determine the role of fast neutral atoms in breakdown of a variety of high power systems. The method can be extended by performing experiments with higher spectral and spatial resolution, for example by using a laser produced plasma as a backlighter to supply more photons.

\section{Acknowledgments}

We thank J.P. VanDevender and T.A. Mehlhorn for valuable technical discussions. Our thanks to P. Menge, D. Hanson, B. Fowler, and the SABRE operations crew for assistance with the experiments. T.A. Mehlhom, C. Olson, and J.P. Quintenz have provided ongoing support and encouragement. Sandia is a multiprogram laboratory operated by Sandia Corporation, a Lockheed Martin Company, for the U.S. Department of Energy under Contract DE-AC04-94AL85000. 


\section{References}

1. D. S. Prono et al, J. Appl. Phys. 52, 3004 (1981).

2. J. P. VanDevender, "Power Flow for Vacuum Insulated Inductive Loads", in Proc. 3rd IEEE Pulsed Power Conference, Albuquerque, NM, June 1-3, 1981, p. 248.

3 R. Pal and D. Hammer, Phys. Rev. Lett. 50, 732 (1983).

4 Y. Maron et al, in Proc. 5th Int. Conf. on High Power Beams, San Francisco, 1983, p. 135; Y. Maron et al, Phys. Rev. Lett. 57, 699 (1986); Y. Maron et al, Phys. Rev. A 39, 5842 (1989).

5. C. Litwin and Y. Maron, Phys. Fluids B 1, 670 (1989).

6. H. Bluhm et al, in Proc. 4th Int. Top. Conf. on High Power Electron and Ion Beam Research and Technology, Palaiseau, France, 1981, p. 87.

7. K. Horioka, N. Tazima, and K. Kasuya, Rev. Sci. Instr. 61, 610 (1990).

8. M. Tuszewski, W. J. Waganaar, and M. P. Desjarlais, J. Appl. Phys. 77, 6188 (1995).

9. T.A. Mehlhorn et al., in Proc. 10th Int. Conf. on High Power Beams, San Diego, 1994, (NTIS Report PB95-144317) p. 53

10. D.J. Johnson et al.; Laser and Part. Beams 16, 185 (1998).

11. Nuclear Fusion by Inertial Confinement, edited by G. Vellarde, Y. Ronen, and J.M. Martinez-Val (CRC Press, Ann Arbor, 1993)

12. M.E. Cuneo et al., IEEE Trans. on Plasma Sci. 25, 229 (1997).

13. T. D. Pointon, J. Appl. Phys. 66, 2879 (1989).

14. M. P. Desjarlais, J. Appl. Phys. 66, 2888 (1989).

15. T. Green Sandia National Laboratory Internal Report SAND95-1794, available from NTIS

16. D. R. Welch and M. E. Cuneo, in Proc. 10th IEEE Pulsed Power Conference, Albuquerque NM, July 10-13,1995; D.R. Welch; D.R. Welch et al., Phys. Plasmas 3, 2113 (1996). 
17. M. E. Cuneo et al, in Proc. 10th IEEE Pulsed Power Conference, Albuquerque NM, July 10-13,1995.

18.R.A. Vesey et al., Phys. Plasmas 6, 3369 (1999).

19 J.E. Bailey et al Phys. Rev. Lett. 74, 1771 (1995).

20. A.B. Filuk et al., Phys. Rev. Lett. 77, 3557 (1996).

21. T. Nash, D. Noack, and A. B. Filuk, Rev. Sci. Instr. 64, 2493 (1993).

22. J. Romand, G. Balloffet, and B. Vodar, Compt. Rend. Acad. Sci. (Paris) 240, 412 (1955). Also: H. Damany, J.-Y. Roncin, and N. Damany-Astoin, Appl. Optics 5, 297 (1966).

23. A.B. Filuk, T.J. Nash, and D.D. Noack, in Proc. 10th Int. Conf. on High Power Beams, San Diego, 1994, (NTIS Report PB95-144317) p. 414

24. A. N. Zaidel' and E. Ya. Shreider, Vacuum Ultraviolet Spectroscopy (Ann Ârbor-Humphrey Science Publishers, 1970), Ch. 7.

25. W.F. Chan, G. Cooper, and C.E. Brion, Chem. Physics 178, 401 (1993).

26. A. Mitchell and M. Zemansky, Resonance Radiation and Excited Atoms (Cambridge Univ. Press, 1971), Ch. 3.

27. W. Lotz, Astrophys. J. Suppl. Series 14, 207(1967).)

28. D.R. Welch, "Gas Breakdown Effects in the PBFA II Diode", Report \#MRC/ABQ-N-553, Mission REsearch Corporation, 1720 Randolph Rd., Albu., N.M., 87106-4245 (1994). 


\section{Tables}

Table 1. Resonance wavelengths and photoionization wavelengths of likely fast atomic neutral atoms

\begin{tabular}{c|cc|cc} 
Neutral & $\begin{array}{c}\text { Resonance } \\
\lambda(\AA)\end{array}$ & $\begin{array}{c}\text { Abs. Oscillator } \\
\text { Strength } f_{\text {abs }}\end{array}$ & $\begin{array}{c}\text { Photoionization } \\
\lambda(\AA)\end{array}$ & $\begin{array}{c}\text { Blue-side Absorption } \\
\text { Coefficient } \mu_{<}\left(\mathrm{cm}^{-1}\right)\end{array}$ \\
\hline H & 1215 & 0.42 & 912 & 213 \\
C & 945 & 0.27 & 1101 & - \\
N & 1200 & 0.35 & 853 & 240 \\
O & 989 & 0.056 & 911 & 121 \\
F & $\sim 955$. & $0.088^{*}$ & 712 & - \\
*Provided by E. J. McGuire, Sandia & &
\end{tabular}

Table 2. Measured upper bounds on fast neutral densities in SABRE AK gap based on absence of absorption lines.

\begin{tabular}{|c|c|c|}
\hline Neutral & & $\begin{array}{c}N_{\text {max from abs. line }} \\
\left(10^{13} \mathrm{~cm}^{-3}\right)\end{array}$ \\
\hline $\mathrm{H}$ & & 1.2 \\
\hline$C$ & & 3.5 \\
\hline$N$ & & 1.6 \\
\hline 0 & $\%$ & 15 \\
\hline$F$ & & 10 \\
\hline
\end{tabular}

Table 3. Measured upper bounds on various molecular densities in SABRE AK gap based on absence of strong absorption bands.

\begin{tabular}{cccc} 
Molecule & Band Center $(\AA)$ & $\mu_{\text {peak }}\left(\mathrm{cm}^{-1}\right)$ & $\mathrm{N}_{\max }\left(10^{14} \mathrm{~cm}^{-3}\right)$ \\
\hline $\mathrm{H}_{2}$ & 839 & 700 & 10. \\
$\mathrm{~N}_{2}$ & 973 & 4500 & 1.6 \\
$\mathrm{O}_{2}$ & 957 & 1650 & 4.3 \\
$\mathrm{CO}_{2}$ & 1125 & 4000 & 1.8 \\
$\mathrm{CO}$ & 932 & 4400 & 1.6 \\
$\mathrm{H}_{2} \mathrm{O}$ & 1030 & 600 & 12.
\end{tabular}


Figure Captions:

Figure 1. Top (a) and side (b) views of VUV line-of-sight in SABRE AK gap (not to scale). In (b) the absorption collection is indicated by solid lines and the emission by dashed lines.

Figure 2. Absorption spectrum (solid) with $5.5 \mathrm{mTorr} \mathrm{CO}_{2}$ differentially-pumped gas fill. The un-absorbed BRV spectrum is shown as a dashed line. Also shown is spectrum (dotdashed) calculated using $\mathrm{CO}_{2}$ absorption data of Chan et al [25].

Figure 3. Relative cathode current (dB/dt monitor) and ion beam current (Faraday cup) histories for SABRE LiF shot \#1247, along with BRV x-ray diode monitor history. VUV spectrometer frame times (arrows) and approximate duration of backlighter VUV output are indicated.

Figure 4. Upper bounds on ground state densities for various atoms as a function of Doppler broadening effective temperature $T_{D}$. The vertical dashed line corresponds to Doppler broadening measured for fast Li I in Ref. 19. 


\section{Tables}

Table 1. Resonance wavelengths and photoionization wavelengths of likely fast atomic neutral atoms

\begin{tabular}{c|cc|cc} 
Neutral & $\begin{array}{c}\text { Resonance } \\
\lambda(\AA)\end{array}$ & $\begin{array}{c}\text { Abs. Oscillator } \\
\text { Strength } \mathrm{f}_{\text {abs }}\end{array}$ & $\begin{array}{c}\text { Photoionization } \\
\lambda(\AA)\end{array}$ & $\begin{array}{c}\text { Blue-side Absorption } \\
\text { Coefficient } \mu_{<}\left(\mathrm{cm}^{-1}\right)\end{array}$ \\
\hline H & 1215 & 0.42 & 912 & 213 \\
C & 945 & 0.27 & 1101 & - \\
N & 1200 & 0.35 & 853 & 240 \\
O & 989 & 0.056 & 911 & 121 \\
F & -955 & $0.088^{*}$ & 712 & - \\
*Provided by E. J. McGuire, Sandia & & &
\end{tabular}

Table 2. Measured upper bounds on fast neutral densities in SABRE AK gap based on absence of absorption lines or edges.

\begin{tabular}{|c|c|c|}
\hline Neutral & $\begin{array}{c}N_{\max } \text { from abs. line } \\
\left(10^{13} \mathrm{~cm}^{-3}\right)\end{array}$ & $\begin{array}{c}N_{\max } \text { from abs. edge } \\
\left(10^{15} \mathrm{~cm}^{-3}\right)\end{array}$ \\
\hline $\mathrm{H}$ & 1.2 & 12.3 \\
\hline C & 5.1 & - \\
\hline $\mathbf{N}$ & 2.1 & 2.9 \\
\hline 0 & 26 & 3.6 \\
\hline$F$ & 20 & - \\
\hline
\end{tabular}

Table 3. Measured upper bounds on various molecular densities in SABRE AK gap based on absence of strong absorption bands.

\begin{tabular}{cccc} 
Molecule & Band Center $(\AA)$ & $\mu_{\text {peak }}\left(\mathrm{cm}^{-1}\right)$ & $N_{\max }\left(10^{14} \mathrm{~cm}^{-3}\right)$ \\
\hline $\mathrm{H}_{2}$ & 839 & 700 & 10. \\
$\mathrm{~N}_{2}$ & 973 & 4500 & 1.6 \\
$\mathrm{O}_{2}$ & 957 & 1650 & 4.3 \\
$\mathrm{CO}_{2}$ & 1125 & 4000 & 1.8 \\
$\mathrm{CO}$ & 932 & 4400 & 1.6 \\
$\mathrm{H}_{2} \mathrm{O}$ & 1030 & 600 & 12.
\end{tabular}




\section{Figure Captions:}

Figure 1. Top (a) and side (b) views of VUV line-of-sight in SABRE AK gap (not to scale). In (b) the absorption collection is indicated by solid lines and the emission by dashed lines.

Figure 2. Unabsorbed (dashed), and absorbed (solid) spectrum with $5.5 \mathrm{mTorr} \mathrm{CO}_{2}$ differentially-pumped gas fill. Also shown is spectrum (dot-dashed) calculated using $\mathrm{CO}_{2}$ absorption data of Chan et al [24].

Figure 3. Relative cathode current ( $\mathrm{dB} / \mathrm{dt}$ monitor) and ion beam (Faraday cup) histories for SABRE LiF shot \#1247, along with BRV $x$-ray diode monitor history. VUV spectrometer frame times (arrows) and approximate duration of backlighter VUV output are indicated.

Figure 4. Upper bounds on ground state densities for various atoms as a function of Doppler broadening effective temperature $T_{D}$. Solid lines indicate true upper bounds, dotted lines show upper bounds at fixed averaging interval $\Delta \lambda_{\mathrm{i}}=2 \AA$. Vertical dashed line corresponds to Doppler broadening expected from 5 mrad equivalent source divergence. 


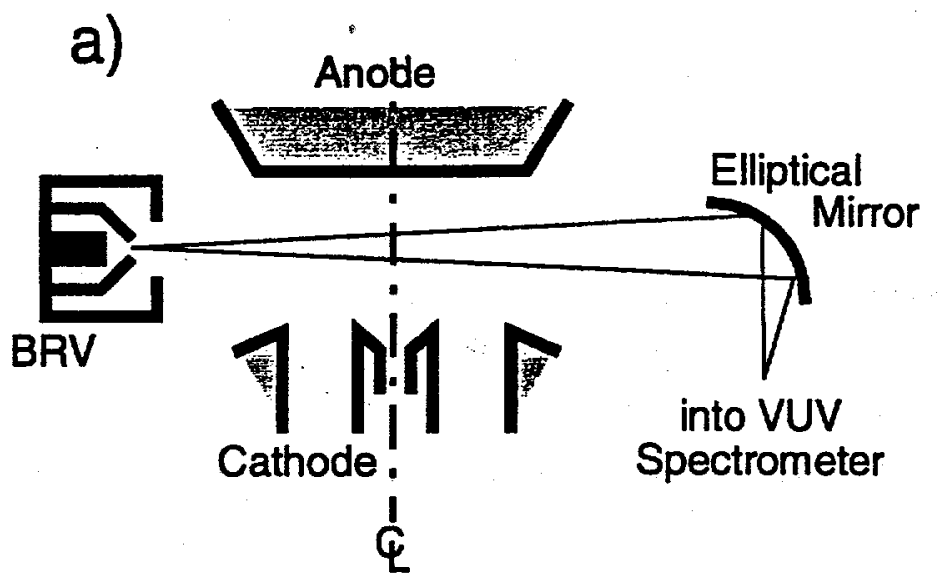

b)

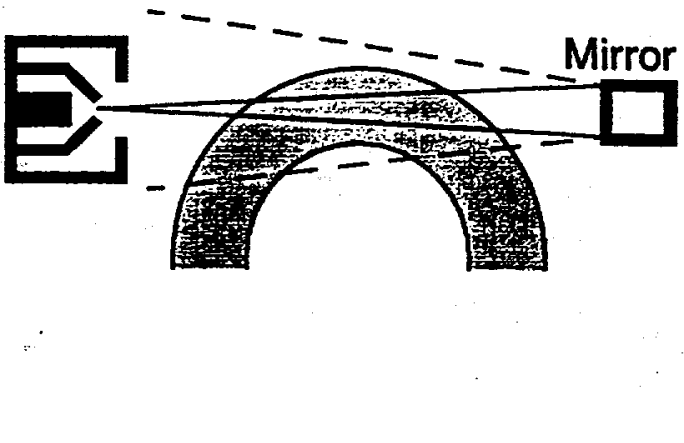


Figure 2

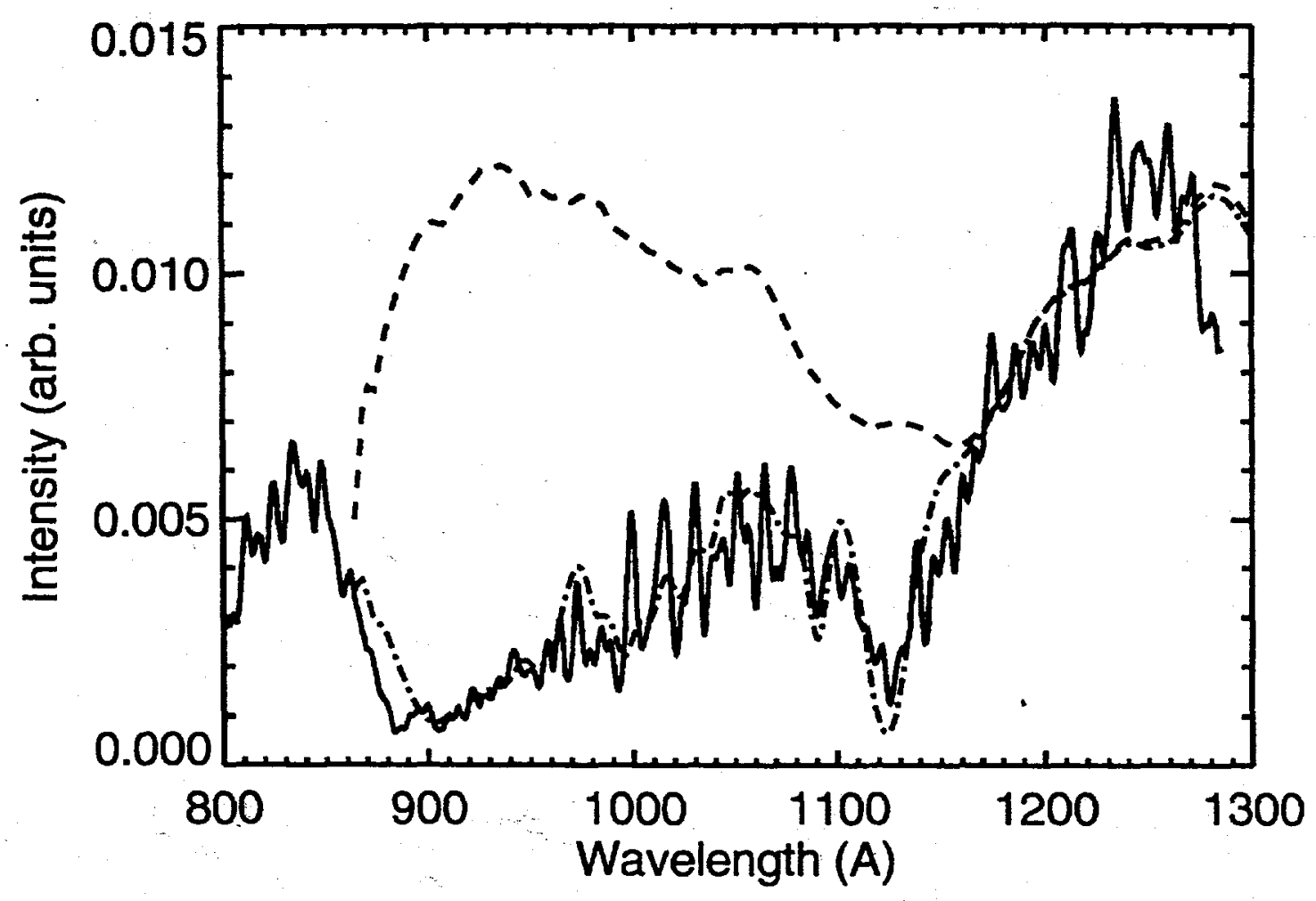


Figure 3

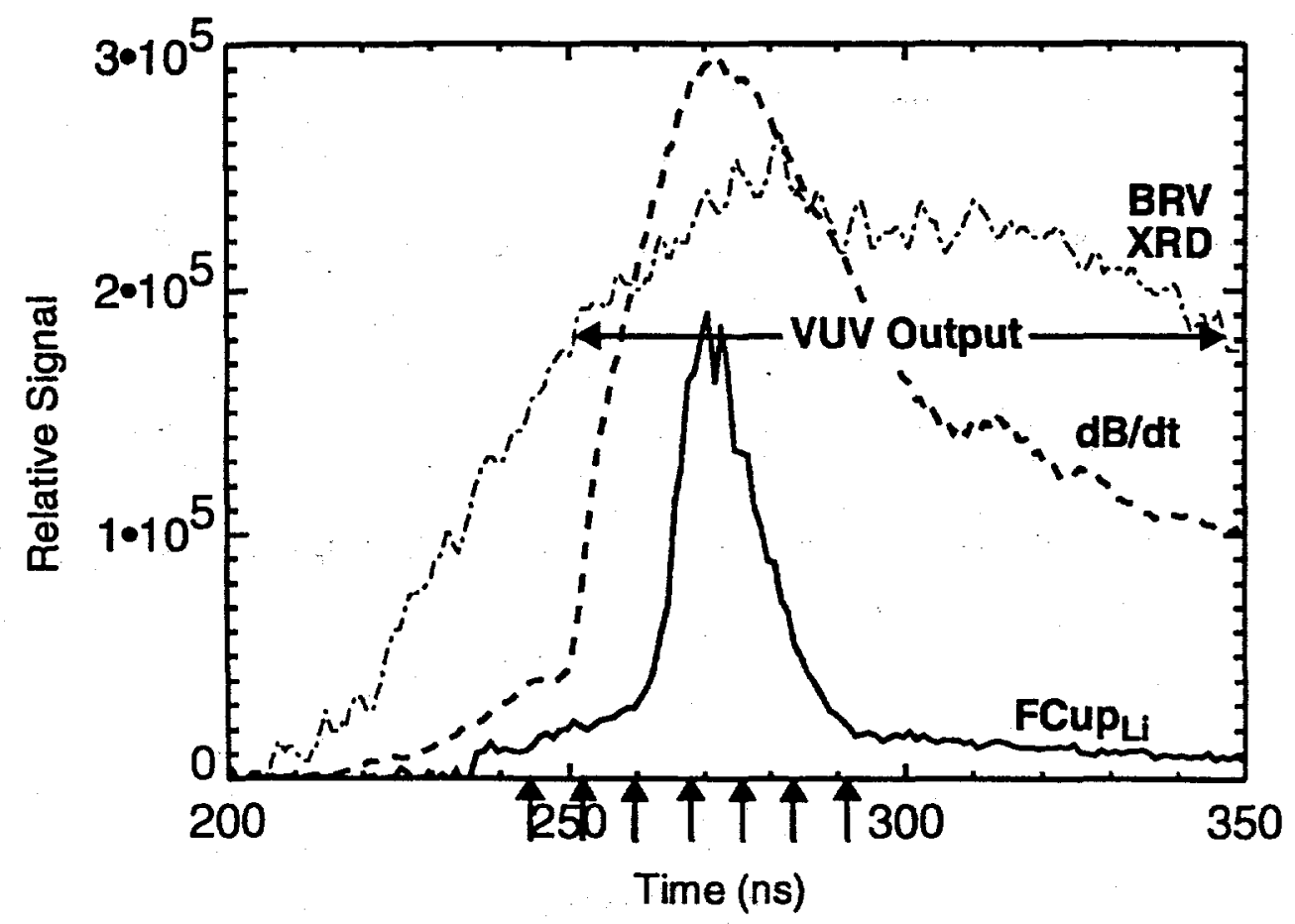


Figure 4

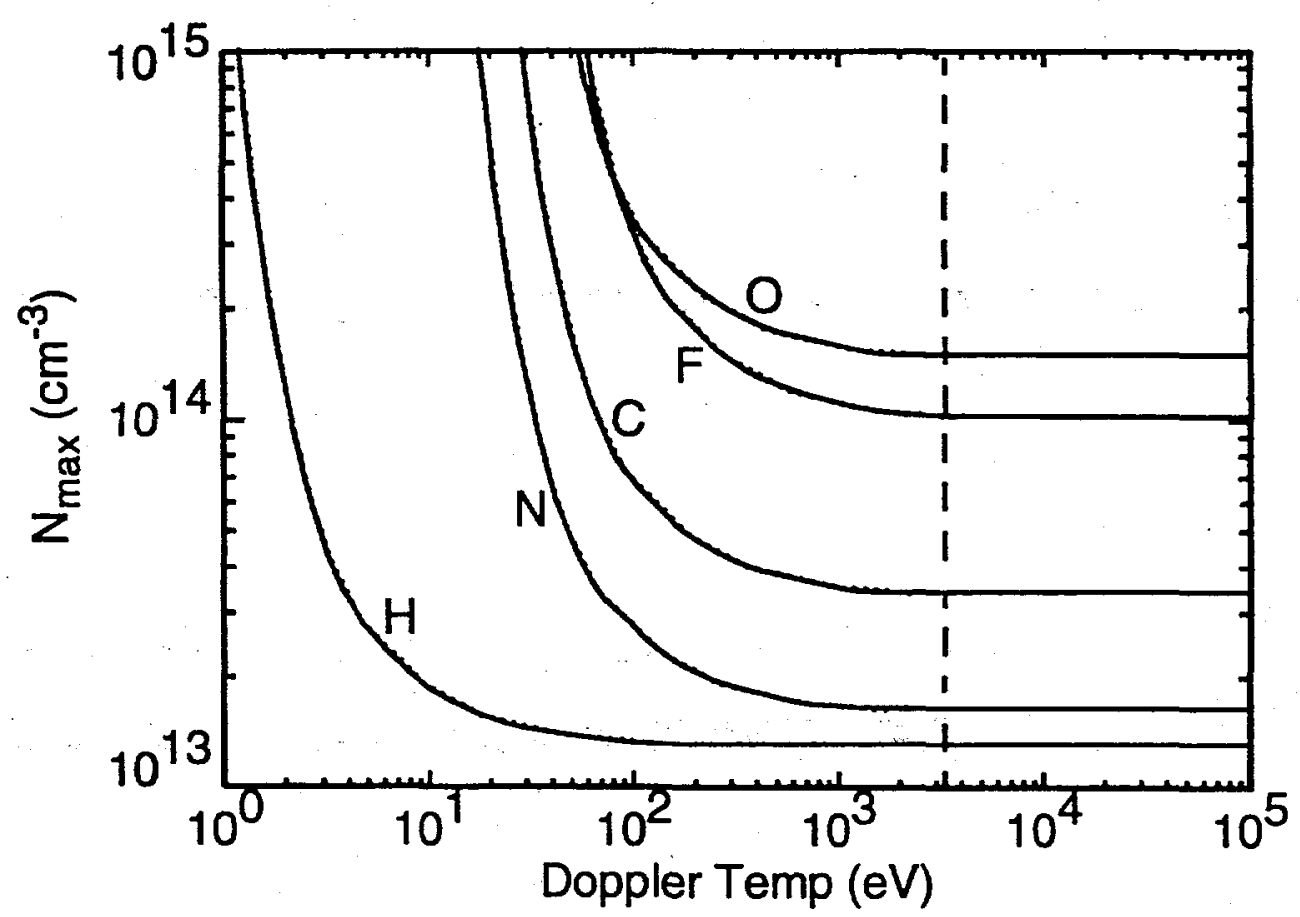

